\title{
Suboptimal therapy controls clinically apparent disease but not subclinical progression of Vogt-Koyanagi-Harada disease
}

\author{
Tatsushi Kawaguchi $\cdot$ Shintaro Horie $\cdot$ \\ Nadia Bouchenaki · Kyoko Ohno-Matsui • \\ Manabu Mochizuki · Carl P. Herbort
}

Received: 20 October 2008/Accepted: 30 December 2008/Published online: 17 January 2009

(C) Springer Science+Business Media B.V. 2009

\begin{abstract}
Purpose To evaluate clinical and angiographic differences in patients with Vogt-KoyanagiHarada (VKH) disease during the early 4-month treatment phase with high- or medium-dose systemic corticosteroid therapy. Methods VKH patients treated at the Centre for Ophthalmic Specialized Care, Lausanne, Switzerland $(n=4)$, or the Department of Ophthalmology, Tokyo Medical and Dental University, Tokyo, Japan $(n=5)$, underwent a pre-treatment indocyanine green angiography (ICGA) and a followup ICGA four months after treatment began. Lausanne patients received high-dose, systemic corticosteroid therapy, with or without immunosuppressive therapy.
\end{abstract}

T. Kawaguchi · S. Horie · K. Ohno-Matsui ·

M. Mochizuki · C. P. Herbort

Department of Ophthalmology and Visual Science,

Tokyo Medical and Dental University Graduate

School of Medicine, 1-5-45 Yushima, Bunkyo-ku,

Tokyo 113-8519, Japan

N. Bouchenaki

Inflammatory and Retinal Eye Diseases, Centre for Ophthalmic Specialized Care, La Source, Lausanne, Switzerland

C. P. Herbort $(\square)$

Inflammatory and Retinal Eye Diseases, Centre for Ophthalmic Specialized Care and University of Lausanne,

6, Rue de la Grotte, CH-1003 Lausanne, Switzerland

e-mail: carl.herb@bluewin.ch
Tokyo patients received medium-dose systemic corticosteroid therapy that included 3 days of intravenous pulse methylprednisolone. ICGA signs including choroidal stromal vessel hyperfluorescence and leakage, hypofluorescent dark dots (HDD), fuzzy vascular pattern of large stromal vessels and disc hyperfluorescence were retrospectively compared. Results The pretreatment ICGA demonstrated that each of the nine patients had choroidal inflammatory foci, as indicated by HDD. At 4-month follow-up, clinical and fluorescein findings had improved almost equally in both groups. HDD had resolved in the Lausanne group but persisted in the Tokyo group. Sunset glow fundus occurred in three of the Tokyo patients and none of the Lausanne patients. Conclusions Submaximal doses of inflammation suppressive therapy are sufficient to suppress clinically apparent disease but not the underlying lesion process. This explains the propensity for sunset glow fundus in seemingly controlled disease.

Keywords Choroiditis - Indocyanine green angiography - Sunset glow fundus .

Vogt-Koyanagi-Harada disease

\section{Introduction}

Vogt-Koyanagi-Harada (VKH) disease is a bilateral granulomatous uveitis that initially presents as a panuveitis or more frequently as a predominantly posterior uveitis with papillitis and exudative retinal 
detachments. There is increasing evidence that the disease is an autoimmune Th1 type reaction directed against proteins related to stromal choroidal melanocytes [1]. Therefore, the primary inflammatory events occur at the level of the choroidal stroma, making it a primary stromal choroiditis [2]. The inflammatory reaction in VKH is sometimes mild and remains subclinical and confined to the choroid, and diagnosis is difficult without sensitive investigational tests of the choroid. Most of the time, however, the inflammatory reaction is hyperacute going beyond the choroid and involving secondarily adjacent structures such as the retinal pigment epithelium and the retina. Although these structures are involved only as a consequence of primary choroidal inflammation, they are accessible to clinical examination and will render the disease clinically apparent, showing the classical signs of multiple exudative retinal detachments and papillitis. These clinical signs respond to massive inflammation suppressive therapy, which is agreed to be necessary to control the hyperacute phase of the disease [3-5].

A consistent finding in a large portion of $\mathrm{VKH}$ patients is the development of sunset glow fundus, even after the clinical disease appears to be under control $[6,7]$. Development of sunset glow fundus in the absence of manifest clinical disease suggests a silently progressing disease and indicates that the post-acute treatment regimen may not be optimal. Until indocyanine green angiography (ICGA) became available, there were no methods sensitive enough to detect subtle choroidal inflammation, and ultrasonography was the imaging method used for VKH disease [8]. However, in order to detect choroidal involvement with ultrasonography, a certain degree of choroidal thickening is necessary, which is the case in established VKH but not in subclinical or early stages of the disease. Ultrasonography is not sensitive enough to detect early and isolated choroidal foci (granuloma) or reappearance of foci in subclinical recurrent VKH. In contrast, ICGA is sensitive enough to detect small choroidal inflammatory foci and to provide information about the choriocapillaris circulation and inflammation of choroidal stromal vessels [9]. Thus, ICGA is currently the most appropriate method for investigating choroidal diseases, as it is sensitive enough to demonstrate minimal and, often, subclinical changes in the choroid [10-12]. Moreover, in cases of suspected VKH disease that do not have an acute onset or that are seen at a later stage, when suboptimal therapy has already been introduced, or that do not exhibit a complete set of signs, diagnosis of VKH becomes more difficult, and the diagnostic contribution of ICGA is especially useful [13-17].

In the present study, we compared VKH patients from two treatment centres where the standard of care differed significantly. Early therapeutic interventions (first week) were comparable in both centres and controlled acute clinical disease. However, after the early phase of therapy (first week), treatment strategies differed markedly in the two centres. At one centre, therapy consisted of persistent high oral doses of corticosteroids slowly tapered, with or without adjuvant immunosuppressive therapy; and in the other centre, therapy consisted of medium doses of more rapidly tapered corticosteroids. We compared ICGA results at presentation and at follow-up after four months of differential therapeutic regimens.

\section{Patients and methods}

\section{Patients}

Patients who were diagnosed with VKH disease according to clinical findings, having presented with an initial acute inflammatory episode between December 1998 and May 2006, had an ICGA before inflammation suppressive therapy was introduced and had an ICGA at follow-up after 4 months of treatment at the Centre for Ophthalmic Specialized Care, Lausanne, Switzerland, or the Department of Ophthalmology, Tokyo Medical and Dental University, Tokyo, Japan. The patients who had a history of penetrating ocular trauma, surgery preceding the onset, or clinical or laboratory evidence of other entities of uveitis, were excluded.

Treatment regimens differed significantly in both centres. In Lausanne, high dose inflammation suppressive therapy consisted of pulse intravenous methylprednisolone (500-1,000 mg/day) for 3 days followed by high levels of oral corticosteroid therapy (initial dose $1.0-1.5 \mathrm{mg} / \mathrm{kg} /$ day prednisone) with a slow tapering schedule for 4 months, or oral corticosteroids only, at the same dose and tapering schedule. For patients who had severe inflammatory findings 
such as bullous retinal detachment and swollen optic disc, cyclosporine was added during the first month of the regimen. In Tokyo, treatment consisted of pulse intravenous methylprednisolone (500-1,000 mg/day) for 3 days followed by a lower dose of oral corticosteroids (initial dose $0.6-0.8 \mathrm{mg} / \mathrm{kg} /$ day prednisone) for the following 4 months. Standard ophthalmologic examination, including best corrected visual acuity, slit-lamp examination of the anterior segment, fundus examination with fundus photography, laser flare photometry, and, in cases of suspected ciliary body effusion, ultrasound biomicroscopy, were performed. In addition, a neurological examination that included a spinal tap to search for monopleiocytosis in the cerebrospinal fluid, a hearing examination, and a skin examination were performed.

Fluorescein angiography and ICGA were performed according to a standard protocol prior to introduction of inflammation suppressive therapy and, 4 months after initiation of treatment. ICGA results were correlated with clinical findings, fluorescein angiograph results, and dose of inflammation suppressive therapy. Particular attention was given to four major ICGA signs classically recorded, including early choroidal stromal vessel hyperfluorescence and leakage, hypofluorescent dark dots (HDD), fuzzy pattern of large stromal vessels, and disc hyperfluorescence (see below for details).

Standard ICGA protocol for inflammatory diseases

A standard ICGA protocol was used [18]. A Topcon 50IA (Topcon, Tokyo, Japan) fundus camera coupled to an Imagenet digitizing system was used in Lausanne. In Tokyo, the Topcon 50IA camera was coupled to an analog video system, and images were later digitized using a commercially available digitizing system (WinTV-PVR-2; Hauppauge Computer, Taipeh, Taiwan). The angiography procedure consisted of three phases. The early, 0-3 min phase demonstrates superimposed retinal and choroidal large vessels and incipient exudation of the dye through the choriocapillaris into the choroidal stroma. The intermediate phase, from $\sim 8 \mathrm{~min}$, demonstrates maximum choroidal stromal background indocyanine green (ICG) fluorescence. The late phase, from $\sim 20 \mathrm{~min}$, depending on fundus pigmentation, demonstrates washout of the dye from the general circulation, with the large choroidal vessels appearing dark against the background stromal fluorescence.

\section{ICGA signs}

ICGA signs in VKH disease have been characterized and are currently used for the initial evaluation and follow-up of VKH disease [18-20]. We chose to evaluate ICGA signs that were estimated to be the most consistent, most useful, and easiest to evaluate and record for follow-up purposes, as previously described $[18,21]$. The signs were:

1. Early choroidal stromal vessel hyperfluorescence and leakage, which suggests severe choroidal stromal inflammatory vasculopathy. It appears early in angiography and indicates the severity of the choroidal inflammatory vasculopathy that, in later phases, gives way to diffuse choroidal hyperfluorescence.

2. Hypofluorescent dark dots (HDD) which are thought to correspond to choroidal foci (granuloma), which are the most consistent and easily recorded angiographic signs, allowing semiquantitative assessment of choroidal inflammatory activity. HDD were evaluated using an arbitrary score from 0 to 4 based on the extension and number of dots, as previously described [22].

3. Fuzzy vascular pattern of large stromal vessels; the fuzzy pattern indicates diffuse inflammatory vasculopathy of stromal vessels. Its presence is apparent during the intermediate phase and, in the late phase, gives way to diffuse stromal hyperfluorescence.

4. Disc hyperfluorescence. The optic disc is dark and nonfluorescent in normal ICGA. Disc hyperfluorescence indicates severe disease and usually regresses rapidly with initial induction therapy. Therefore, it is a valuable marker for response to initial high-dose anti-inflammatory therapy.

Statistical analysis

The data are expressed as the mean $\pm \mathrm{SD}$. Data were analyzed and compared using Mann-Whitney U test, and were considered statistically significant with $P<0.05$. 


\section{Results}

Patients, clinical features, and fluorescein angiography results

Nine patients (four in Lausanne, five in Tokyo) fulfilled the inclusion criteria. They underwent ICGA prior to initiation of inflammation suppressive therapy. At least one additional ICGA was performed after 4 months of therapy. In Lausanne, all patients had monthly ICGA for 4 months. Lausanne patients were females. Of the five Tokyo patients, two were female and three were males. The mean age of patients was lower in the Lausanne group (32.0 \pm 6.1 years) compared to the Tokyo group (45.6 \pm 15.1 years), but this difference was not significant. All patients classified as incomplete VKH of the revised diagnostic criteria at presentation, as they also include chronic signs and do not allow a diagnosis of complete VKH at disease onset [23]. Patient and disease characteristics are shown in Table 1. The groups did not differ significantly on any variables at presentation. For all patients, the inflammatory episode marked the onset of VKH. The disease was bilateral with papillitis and lymphopleiocytosis in the cerebrospinal fluid for all nine patients, and multiple exudative retinal detachments were present in three of the Lausanne patients and four of the Tokyo patients. Uveitis had an initial anterior component and presented as a panuveitis in three Lausanne patients and four Tokyo patients. Fluorescein angiographs in the Lausanne group were characterized by bright disc hyperfluorescence in all four patients (8 eyes) and signs of exudative retinal detachments and subretinal fluid in three patients (5 eyes). In the Tokyo group, disc hyperfluorescence was observed in five patients (10 eyes), and exudative retinal detachments with pooling of subretinal fluid was observed in four patients ( 8 eyes). The delay from the onset of symptoms to initiation of initial high-dose inflammation suppressive therapy was evaluated in both groups, and the mean delay was $4.5 \pm 3.1$ weeks in Lausanne patients and $2.4 \pm 0.6$ weeks in the Tokyo patients, indicating that diagnostic delay is longer in countries where VKH occurs sporadically, but this difference was not significant. Intravenous pulse methylprednisolone therapy (500-1,000 mg/day for 3 days) was administered in two Lausanne and five Tokyo patients. Mean oral averaged prednisone dose $/ \mathrm{kg} /$ day during the 4-month treatment period was significantly higher $(0.75 \pm 0.2 \mathrm{mg} / \mathrm{kg} / \mathrm{day} ;$ initial dose $1.0-1.5 \mathrm{mg} / \mathrm{kg} /$ day) for the Lausanne group compared to the Tokyo group $(0.36 \pm 0.02 \mathrm{mg} / \mathrm{kg} /$ day; initial dose $0.6-$ $0.8 \mathrm{mg} / \mathrm{kg} / \mathrm{day} ; p=0.039)$. Two of the four Lausanne patients also received cyclosporine therapy. The mean averaged daily dose per month was $1.04 \pm 0.37 \mathrm{mg} / \mathrm{kg} /$ day for the Lausanne group versus $0.52 \pm 0.07 \mathrm{mg} / \mathrm{kg} /$ day for the Tokyo group for the first month and $0.55 \pm 0.15 \mathrm{mg} / \mathrm{kg} /$ day versus
Table 1 Demographics and disease characteristics in the Lausanne and Tokyo groups

\begin{tabular}{lll}
\hline & Lausanne & Tokyo \\
\hline Sex & $4 \mathrm{~F}$ & $2 \mathrm{~F}, 3 \mathrm{M}$ \\
Mean age & $32.0 \pm 6.1$ years & $45.6 \pm 15.1$ \\
Bilaterality & $4 / 4$ patients & $5 / 5$ patients \\
CSF monopleiocytosis & $4 / 4$ patients & $5 / 5$ patients \\
Hearing disorders & $0 / 4$ patients & $1 / 5$ patients \\
Time from symptoms to treatment & $4.5 \pm 3.1$ weeks & $2.4 \pm 0.6$ weeks \\
Initial anterior uveitis & $3 / 4$ patients & $4 / 5$ patients \\
Exudative retinal detachments & $3 / 4$ patients & $4 / 5$ patients \\
Papillitis & $4 / 4$ patients & $5 / 5$ patients \\
Sunset glow fundus at 4 months & $0 / 4$ patients & $3 / 5$ patients \\
Integumentory findings at 4 months & $0 / 4$ patients & $1 / 5$ patients \\
VAOD initial & $0.80 \pm 0.28$ & $0.94 \pm 0.36$ \\
VAOS initial & $0.77 \pm 0.29$ & $0.90 \pm 0.24$ \\
VAOD final & $1.44 \pm 0.12$ & $1.44 \pm 0.12$ \\
VAOS final & $1.31 \pm 0.24$ & $1.44 \pm 0.12$ \\
\hline
\end{tabular}




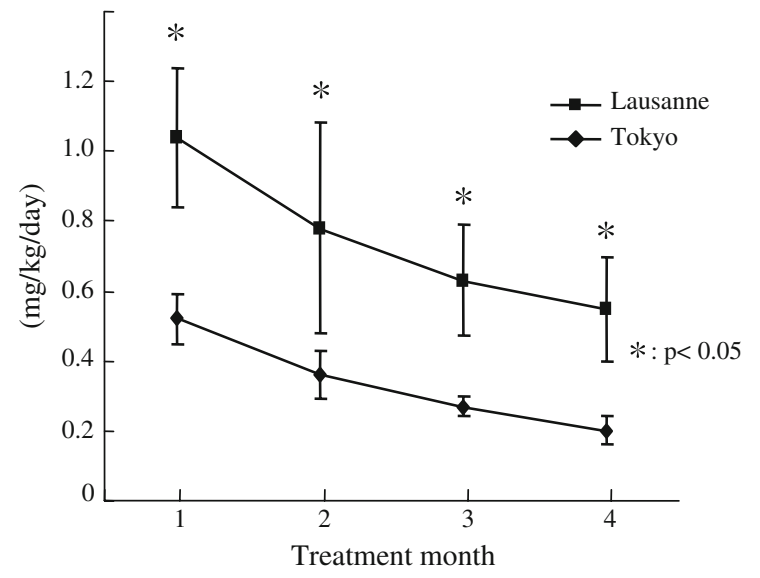

Fig. 1 Graph showing chronology of the two oral prednisone treatment regimens

$0.20 \pm 0.04$ for the fourth month, respectively. Details of treatment regimen during the 4-month period are shown in Fig. 1.

Mean best corrected visual acuity for Lausanne patients was $0.78 \pm 0.3$ ( 8 eyes) at presentation and $1.37 \pm 0.18$ ( 8 eyes) after 4 months. Values for the Tokyo patients were $0.92 \pm 0.32$ at presentation and $1.44 \pm 0.12$ (10 eyes) at 4 months, indicating that both groups had complete visual recovery after 4 months. Fundus lesions recovered and fluorescein angiography signs were considered normal in all nine patients at 4 months. However, at 4 months, three of the five Tokyo patients had mild to moderate sunset glow fundus. Sunset glow fundus did not occur in any of the four Lausanne patients.

One of the Tokyo patients developed a hyperglycemia after corticosteroid therapy was initiated, but the serum glucose level was decreased within normal limits by a diet. None of the Lausanne patients showed any side effects of inflammation suppressive therapy during observation.

\section{ICGA data}

All the patients had consistent ICGA findings at presentation. Early hyperfluorescent leaking stromal vessels were seen in three of the Lausanne patients, and the mean time to resolution of this sign was $1.88 \pm 0.76$ months. The sign was not detected in any of those patients at 4 months. All five Tokyo patients showed this ICGA sign at presentation, with a larger number of vessels involved compared to the
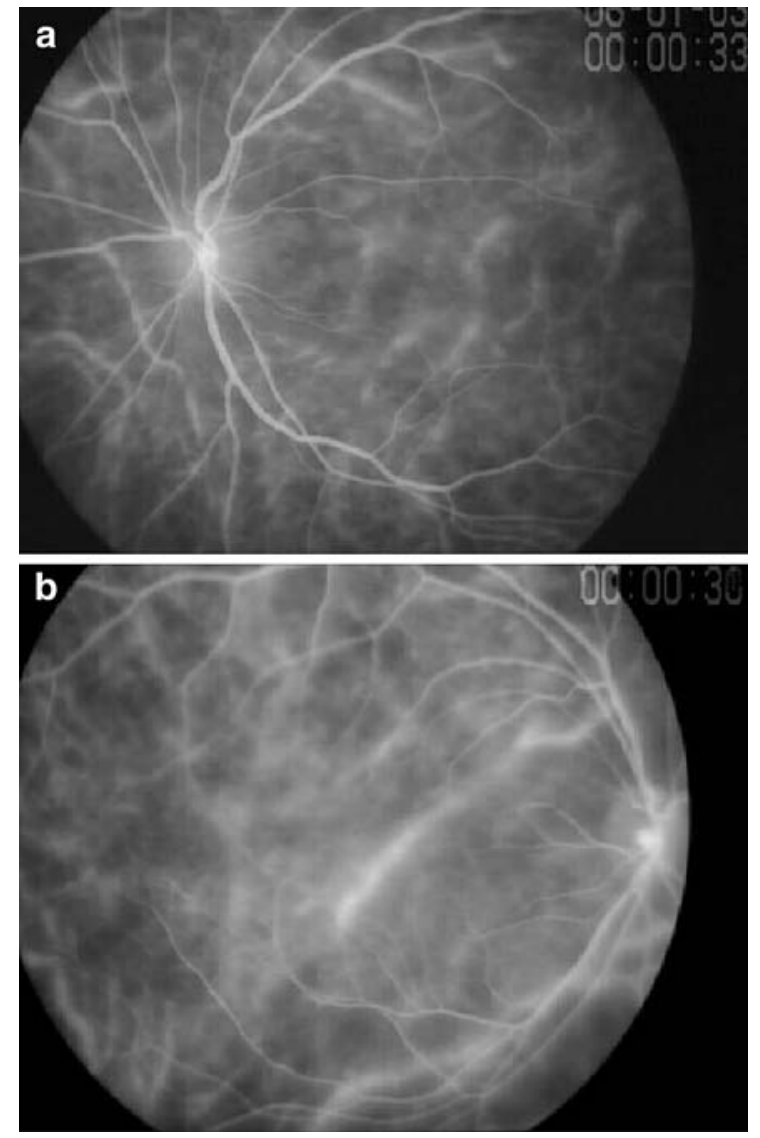

Fig. 2 Early hyperfluorescent leaking stromal vessels. Numerous hyperfluorescent posterior pole stromal vessels prior to treatment (a). For two Japanese patients, this sign persisted after 4 months of treatment (b)

Lausanne group (Fig. 2a). At the 4-month ICGA follow-up, the sign was still present in two of the Tokyo patients (Fig. 2b).

HDD were semi-quantitatively evaluated with an arbitrary score from 0 to 4 . HDD intensity and score were similar for the two groups at presentation in the ICGA intermediate phase $(3.5 \pm 0.53$ for the Lausanne group and $3.7 \pm 0.42$ for the Tokyo group; Fig. 3a, c). At 4 months, HDD was more than $90 \%$ resolved in all patients of the Lausanne group, with a mean time to resolution of $2.62 \pm 1.25$ months and a decrease in HDD score from $3.5 \pm 0.53$ to $0.25 \pm 0.46$; (Fig. 3b). The situation was different for the Tokyo group, in which HDD persisted at nearly identical intensity and score $(3.7 \pm 0.42$ at presentation and $3.25 \pm 0.46$ at 4 months), although ophthalmic findings improved almost equally in both groups (Fig. 3d). 

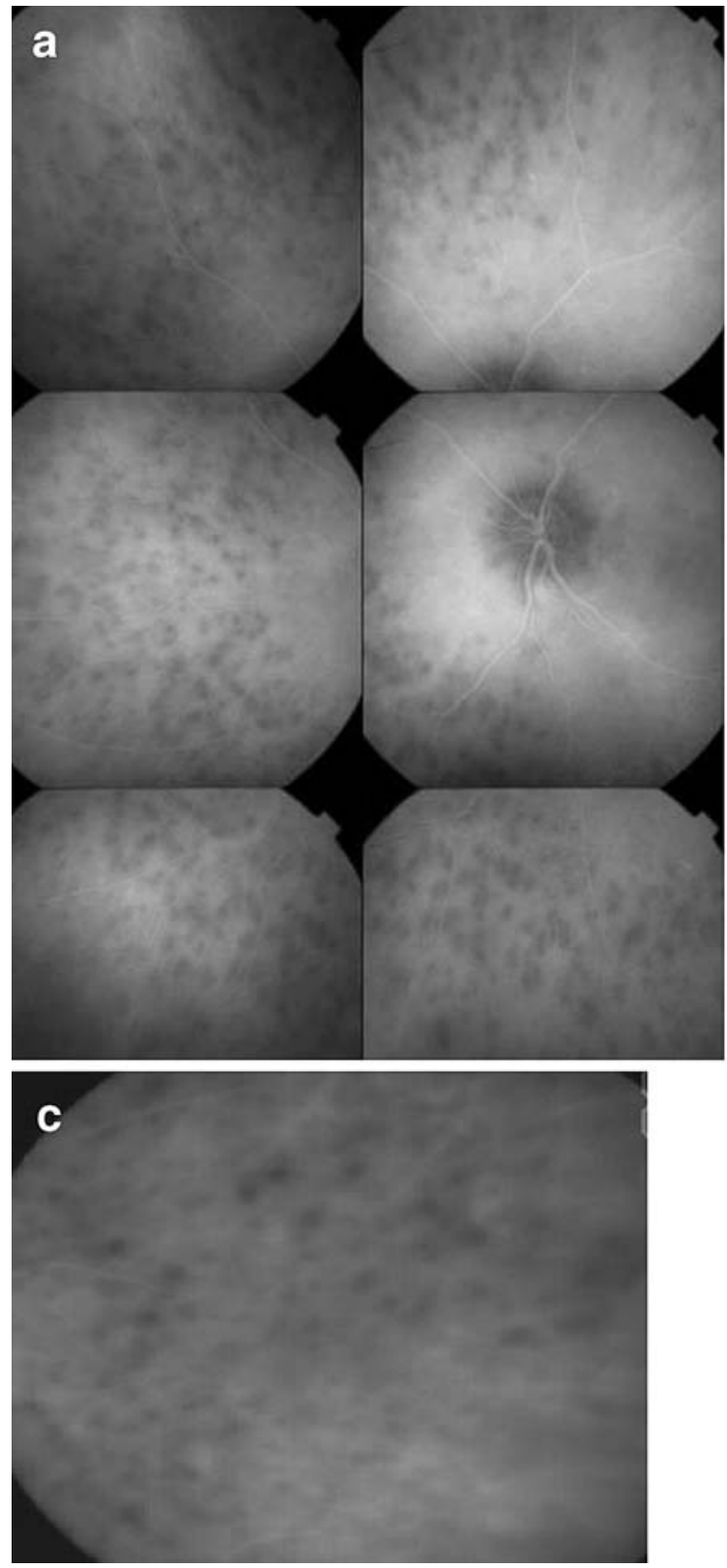

Fig. 3 Hypofluorescent dark dots (HDD). Numerous well demarcated dark spots are seen in the intermediate angiographic phase. Also, note the fuzzy choroidal stromal vessels, the pattern of which cannot be distinguished (a Swiss patient, c

Fuzzy vascular pattern of large stromal vessels was identical in the two groups at presentation. It resolved completely in the Lausanne patients (Fig. 4a, b) at a mean of $2.12 \pm 0.85$ months, and it persisted in the Tokyo patients (Fig. 4c, d). The only ICGA sign that resolved completely in both groups was disc
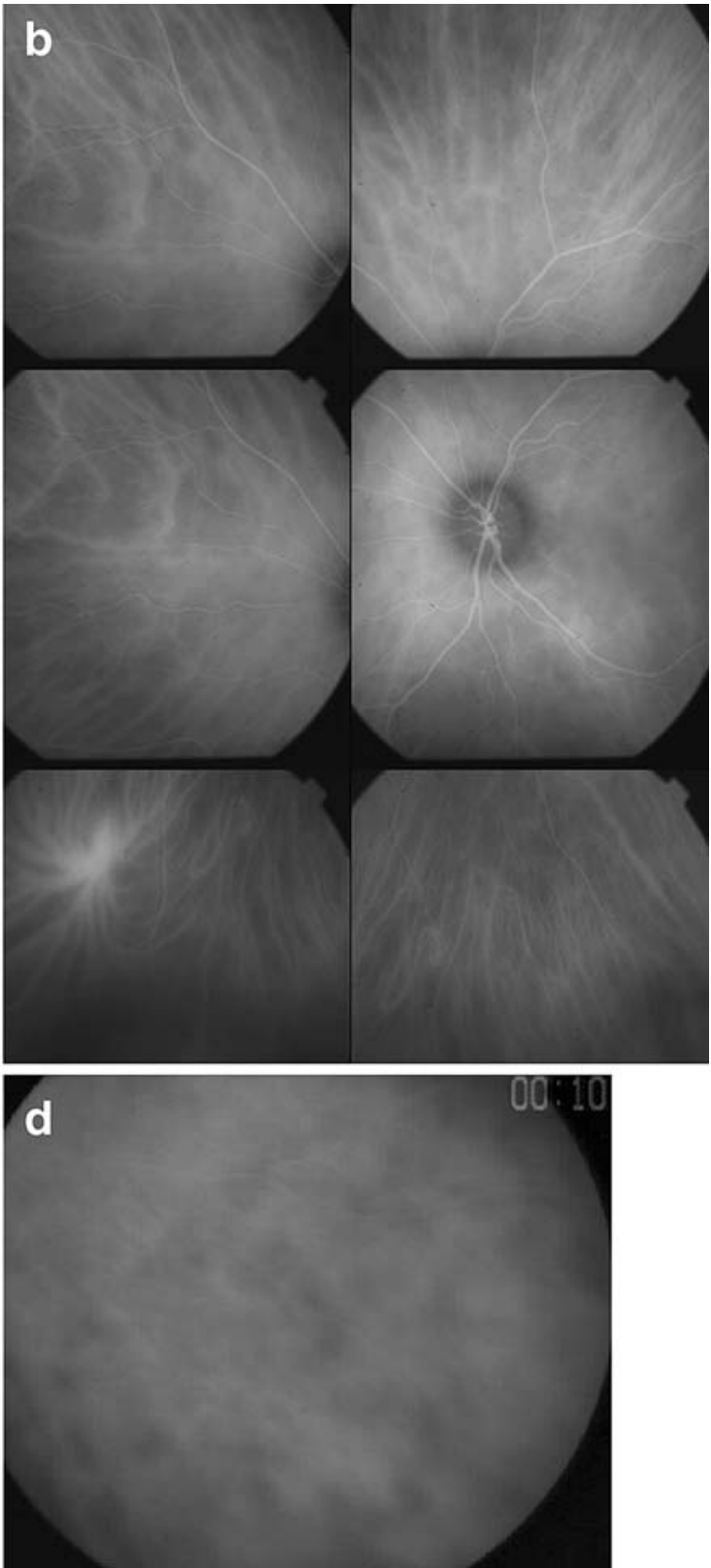

Japanese patient). In Swiss patients, HDD resolved after 1 month of sustained high-dose corticosteroid therapy (b) whereas HDD and fuzzy vessel appearance were persisted in Japanese patients at 4 months after treatment (d)

hyperfluorescence. It was the first ICGA sign to respond to treatment in the Lausanne group, with a mean resolution time of $1.25 \pm 0.86$ months. It was resolved in the Tokyo group at 4 months (Fig. 5a, b).

As mentioned in the "Patients and methods" section, we did not evaluate all ICGA signs. Most 

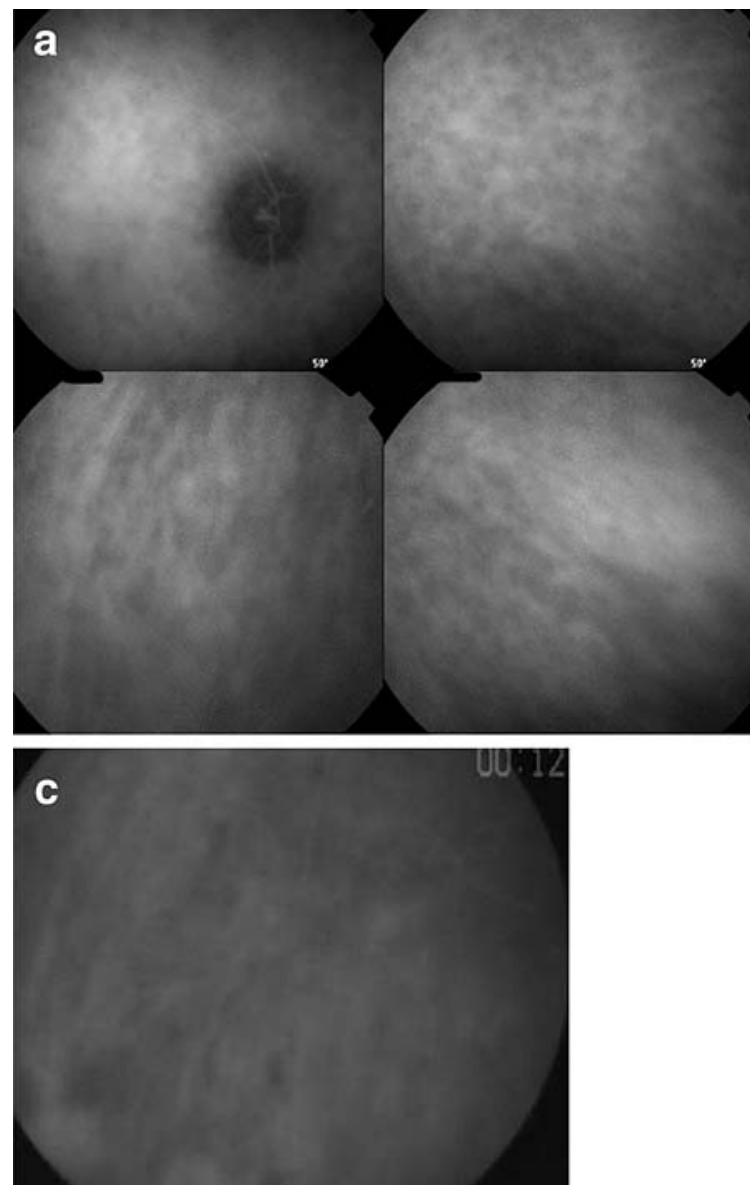

Fig. 4 Fuzzy pattern of larger stromal vessels in a Lausanne patient. Prior to treatment no stromal vessels can be recognized (a Swiss patient, c Japanese patient). After treatment, a normal

patients presented some degree of delay in choroidal filling in the early angiography phase before treatment was initiated.

\section{Discussion}

Onset of VKH disease is characterized by a prodromal stage during which the Th1 autoimmune reaction against melanocyte-associated antigens is believed to cause silently developing lesions within the choroid. When adjacent, functionally important structures, such as the optic disc and the macula are involved, the disease becomes clinically apparent [24]. This phase of VKH disease is typically managed with high-dose pulse intravenous corticosteroids or high-dose oral corticosteroids followed by oral
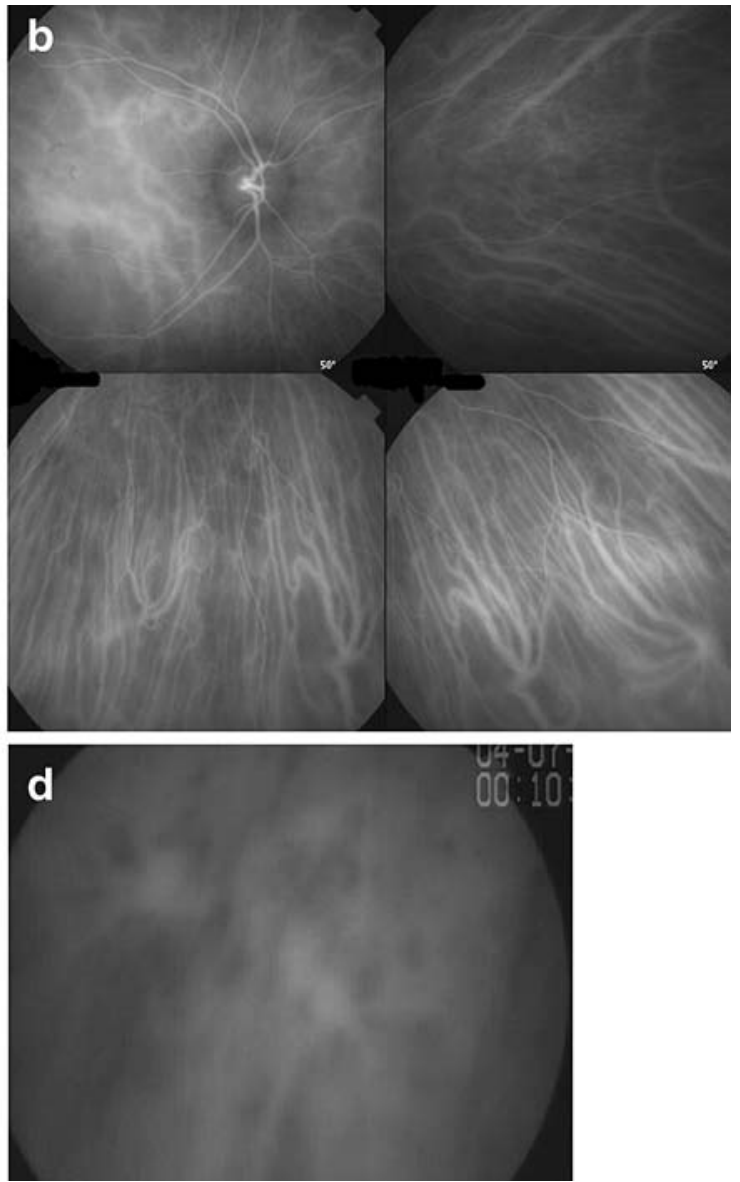

vascular pattern of stromal vessels can be seen in the Swiss patient (b), whereas the situation did not change 4 months after treatment in Japanese patient (d)

corticosteroids [25]. Although that strategy is usually successful in controlling acute disease and restoring visual function, it does not appear to influence longterm outcomes [26]. A recent study that evaluated data from several centres showed that pulse intravenous corticosteroid therapy did not influence longterm outcome of the disease [27].

Final outcomes for VKH disease seem to depend on long-term treatment strategies. To date, long-term management of VKH disease is not clearly defined and varies across centres and cases, including the dose of oral corticosteroids after the initial (first week) highdose inflammation suppressive treatment, the tapering protocol, and the suitability of concomitant immunosuppressive therapy. Data are accumulating which suggest that current long-term management strategies for VKH disease are unsatisfactory, and several lines of 
a

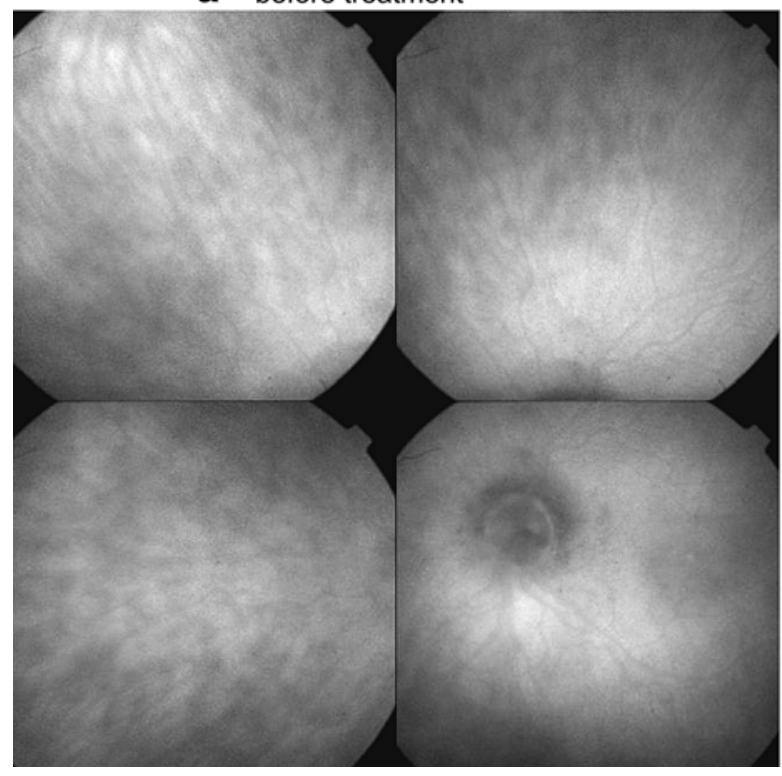

b after treatment

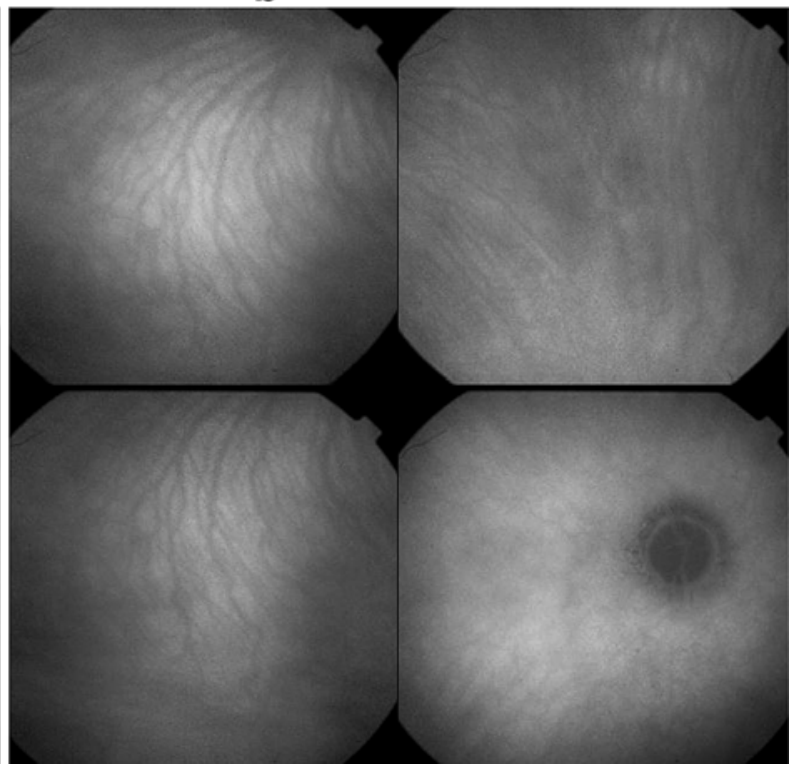

Fig. 5 Disc hyperfluorescence. Prior to treatment, the optic disc is hyperfluoresscent and HDD are present (a). After treatment, the disc is no longer fluorescent and HDD have resolved (b)

evidence indirectly show that the post-acute level of inflammation suppressive therapy is often suboptimal. Sunset glow fundus was shown to develop in most reported series, even in seemingly controlled VKH disease, and is more prominent in VKH disease with clinically persistent inflammatory signs [6, 7, 28, 29]. This suggests that VKH disease progressed despite seemingly sufficient treatment. Our ICGA comparisons of two treatment groups show that choroidal disease was ongoing in patients that received less oral prednisone treatment in the 4 months post-acute treatment period. To the best of our knowledge, ours is the only study to provide direct evidence that submaximal treatment during the post-acute period of VKH disease is associated with persistent choroidal inflammatory activity, despite apparent control of clinical signs. On the other hand, sufficient high-dose post-acute therapy completely suppressed choroidal inflammatory disease within 2-4 months. Our results also demonstrate that clinical examination and fluorescein angiography are not sufficient, but ICGA is required for precise monitoring of inflammatory activity especially at the choroidal level.

A dose of $0.36 \mathrm{mg} / \mathrm{kg} /$ day is not sufficient to suppress all choroidal inflammatory signs and a dose as high as $0.75 \mathrm{mg} / \mathrm{kg} /$ day appears to be required during the first 4 months of treatment. In the present study, we evaluated the effects of inflammation suppressive therapy in four European patients after 4 months of therapy. We determined that the level of therapy necessary to achieve similar control of the disease in Japanese patients may have to be even higher, as the amount of choroidal pigment is probably higher in Japanese patients compared to European patients. However, all the European patients were from southern Europe and had dark complexions. In fact, one of those patients was of American Indian descent. Nevertheless, the present findings justify a controlled, ICGA monitored study comparing Japanese patients receiving standard treatment (i.e., medium-dosed post-acute oral corticosteroid therapy) to Japanese patients with high-dose oral post-acute corticosteroid therapy, with concomitant immunosuppressive therapy when indicated.

The present study did not provide information about an acceptable tapering course. Previously published data show that by monitoring therapy taper with ICGA subclinical choroidal recurrence of VKH disease can be detected [21]. Since ICGA is a sensitive method for investigating choroidal inflammatory lesions, it should probably be the method of 
choice with which to precisely adjust inflammation suppressive therapy in VKH disease.

There is an increasing number of studies that support our finding that deleterious outcomes are associated with insufficient long-term inflammation suppressive therapy $[30,31]$. This is further supported by recent studies which indicate that additional immunosuppressive therapy seems to be beneficial [30, 31]. With these results in mind, it seems advisable to prevent unfavorable outcomes in VKH patients. For this purpose, clinical signs and fluorescein angiography are no longer the only methods for monitoring treatment efficacy. Now, efficacy can be more precisely evaluated with ICGA, allowing preventive therapy adjustments during the tapering phase of treatment [21].

To summarize, suboptimal inflammation suppressive therapy in the post-acute period of VKH disease may be sufficient to suppress clinically apparent disease, but it does not suppress the underlying lesion process, which explains the development of sunset glow fundus in seemingly controlled disease. Based on our findings, we recommend high-dosed, ICGAguided therapy for ocular VKH disease.

\section{References}

1. Sugita S, Takase H, Taguchi C et al (2006) Ocular infiltrating CD4+T cells from patients with Vogt-KoyanagiHarada disease recognize human melanocyte antigens. Invest Ophthalmol Vis Sci 47:2547-2554. doi: 10.1167/iovs.05-1547

2. Bouchenaki N, Herbort CP (2004) Stromal choroiditis. In: Pleyer U, Mondino B (eds) Essentials in ophthalmology: uveitis and immunological disorders. Springer, Berlin, pp 234-253

3. Sonoda S, Nakao K, Ohba N (1999) Extensive chorioretinal atrophy in Vogt-Koyanagi-Harada disease. Jpn J Ophthalmol 43:113-119. doi:10.1016/S0021-5155(98)00066-5

4. Rao NA (2006) Treatment of Vogt-Koyanagi-Harada disease by corticosteroids and immunosuppressive agents. Ocul Immunol Inflamm 14:71-72. doi:10.1080/09273 940600674368

5. Bouchenaki N, Morisod L, Herbort CP (2006) VogtKoyanagi-Harada syndrome: importance of rapid diagnosis and therapeutic intervention. Klin Monatsbl Augenheilkd 216:1-5

6. Keino H, Goto H, Ususi M (2002) Sunset glow fundus in Vogt-Koyanagi-Harada disease with or without chronic inflammation. Graefes Arch Clin Exp Ophthalmol 240:878-882. doi:10.1007/s00417-002-0538-Z
7. Yang $\mathrm{P}$, Wang $\mathrm{H}$, Zhou $\mathrm{H}$ et al (2002) Clinical manifestations and diagnosis of Vogt-Koyanagi-Harada syndrome. Zhonghua Yan Ke Za Zhi 38:736-739

8. Foster DJ, Cano MR, Green RL, Rao NA (1990) Echographic features of the Vogt-Koyanagi-Harada Syndrome. Arch Ophthalmol 108:1421-1426

9. Bouchenaki N, Cimino L, Auer C, Tran VT, Herbort CP (2002) Assessement and classification of choroidal vasculitis in posterior uveitis using indocyanine green angiography. Klin Monatsbl Augenheilkd 219:243-249. doi:10.1055/s-2002-30661

10. Khairallah M, Ben Yahia S, Attia S et al (2006) Indocyanine green angiographic features in multifocal chorioretinitis associated with West Nile virus infection. Retina 26:358359. doi:10.1097/00006982-200603000-00019

11. Machida S, Tanaka M, Murai K, Takahashi T, Tazawa Y (2004) Choroidal circulatory disturbance in ocular sarcoidosis without the appearance of retinal lesions or loss of visual function. Jpn J Ophthalmol 48:392-396. doi: 10.1007/s10384-004-0087-6

12. Howe L, Stanford M, Graham E, Marshall J (1998) Indocyanine green angiography in inflammatory eye diseases. Eye 12:761-767

13. Harada T, Kanbara Y, Takeuchi T, Niwa T, Majima T (1997) Exploration of Vogt-Koyanangi Harada syndrome by infrared choroidal angiography with indocyanine green. Eur J Ophthalmol 7:163-170

14. Oshima Y, Harino S, Hara Y, Tano Y (1996) Indocyanine green angiographic findings in Vogt-Koyanagi-Harada disease. Am J Ophthalmol 122:58-66

15. Okada AA, Mizusawa T, Sakai J, Usui M (1998) Videofunduscopy and videoangiography using the scanning laser ophthalmoscope in Vogt-Koyanagi-Harada disease. Br J Ophthalmol 82:1175-1181

16. Bouchenaki N, Herbort CP (2001) The contribution of indocyanine green angiography to the appraisal and management of Vogt Koyanagi-Harada. Ophthalmology 108:54-64. doi:10.1016/S0161-6420(00)00428-0

17. Bouchenaki N, Herbort CP (2000) Indocyanine green angiography (ICGA) in the assessement and follow-up of choroidal inflammation in active chronically evolving Vogt-Koyanagi-Harada disease. In: Dodds EM, Couto CA (eds) Uveitis in the third millenium. Elsevier, Amsterdam, pp 35-38

18. Herbort CP, LeHoang P, Guex-Crosier Y (1998) Schematic interpretation of indocyanine green angiography in posterior uveitis using a standard protocol. Ophthalmology 105:432-440. doi:10.1016/S0161-6420(98)93024-X

19. Altan-Yaycioglu R, Akova YA, Akca S, Yilmaz G (2006) Inflammation of the posterior uvea: findings on fundus fluorescein and indocyanine green angiography. Ocul Immunol Inflamm 14:171-179. doi:10.1080/09273940600 660524

20. Kohno T, Miki T, Shiraki K et al (1999) Subtraction ICG angiography in Harada's disease. Br J Ophthalmol 83:822833

21. Herbort CP, Mantovani A, Bouchenaki N (2007) Indocyanine green angiography in Vogt-Koyanagi-Harada disease: angiographic signs and utility in patient follow-up. Int Ophthalmol 27:173-182. doi:10.1007/s10792-007-9060-y 
22. Herbort CP, Probst K, Cimino L, Tran VT (2004) Differential inflammatory involvement in retina and choroid in birdshot chorioretinopathy. Klin Monatsbl Augenheilkd 221:351-356. doi:10.1055/s-2004-812827

23. Read RW, Holland GN, Rao NA et al (2001) Revised diagnostic criteria for Vogt-Koyanagi-Harada disease: report of an international committee on nomenclature. Am J Ophthalmol 131:647-652. doi:10.1016/S0002-9394(01)0 0925-4

24. Damico FM, Kiss S, Young LH (2005) Vogt-KoyanagiHarada disease. Semin Ophthalmol 20:183-190. doi: 10.1080/08820530500232126

25. Sasamoto Y, Ohno S, Matsuda H (1990) Studies on corticosteroid therapy in Vogt-Koyanagi-Harada disease. Ohthalmologica 201:162-167

26. Yamanaka E, Ohguro N, Yamamoto S et al (2002) Evaluation of pulse corticosteroid therapy for Vogt-KoyanagiHarada disease assessed by optical coherence tomography. Am J Ophthalmol 134:454-456. doi:10.1016/S0002-9394 (02)01575-1
27. Read RW, Yu F, Accorinti M et al (2006) Evaluation of the effect on outcomes of the route of administration of corticosteroids in acute Vogt-Koyanagi-Harada disease. Am J Ophthalmol 142:119-124. doi:10.1016/j.ajo.2006.02.049

28. Keino H, Goto H, Mori H, Iwasaki T, Ususi M (2006) Association between severity of inflammation in CNS and development of sunset glow fundus in Vogt-KoyanagiHarada disease. Am J Ophthalmol 141:1140-1142. doi: 10.1016/j.ajo.2006.01.017

29. Suzuki S (1999) Quantitative evaluation of "sunset glow" fundus in Vogt-Koyanagi-Harada disease. Jpn J Ophthalmol 43:327-333. doi:10.1016/S0021-5155(99)00016-7

30. Al-Kharashi AS, Aldibhi H, Al Fraykh H, Kangave D, El-Asrar Abu (2007) Prognostic factors in Vogt-KoyanagiHarada disease. Int Ophthalmol 27:201-210. doi: 10.1007/s10792-007-9062-9

31. Bykhovskaya I, Thorne JE, Kempen JH, Dunn JP, Jabs DA (2005) Vogt-Koyanagi-Harada disease: clinical outcomes. Am J Ophthalmol 140:674-678 